\title{
Soil microbial dynamics and enzyme activities as influenced by organic and inorganic nutrient management in vertisols under aerobic rice cultivation
}

Authors Info

S. Jaffar Basha*, R. Basavarajappa, Geeta Shirnalli ${ }^{3}$ and H.B. Babalad'

'Department of Agronomy, College of Agriculture, Dharwad- 580005 , India

${ }^{2}$ Department of Agronomy and Head, Staff Training Unit, Dharwad-580 005, India

${ }^{3}$ Agricultural Microbiology, AICRP on Renewable energy sources, MARS, Dharwad-580 005, India

*Corresponding Author Email : shaik.jaffarbasha@gmail.com

Key words

Aerobic rice,

Chemical fertilizers,

Enzymatic activity,

Microbial population,

Organic manure, Vertisol

Publication Info

Paper received: 10.11 .2015

Revised received : 11.03 .2016

Re-revised received : 13.06 .2016

Accepted : 13.07.2016
Abstract

Aim : An experiment was conducted to determine the effect of organic and inorganic nutrient management practices on dynamics of soil microbial biomass and enzyme activities in vertisols under aerobic rice cultivation during kharif2013 and 2014 at Main Agricultural Research Station, Dharwad.

Methodology : The experiment consisted of twenty four treatment combinations, comprising of six main plots (manurial practices) and four sub plots (liquid organic manures), which were replicated thrice. The seeds of variety MAS-26, developed specifically for aerobic rice production from UAS, Bengaluru were used.

Results : Integrated application of Farm Yard Manure (FYM) (1/3) + vermicompost (1/3) + green leaf manure (1/3) equivalent to Recommended Dose of Nitrogen (RDN) with recommended FYM + microbial consortium (Azospirillum + PSB) with soil application of biodigester @ $2500 \mathrm{I} \mathrm{ha}^{-1}$ at 30,60 and 90 DAS recorded significantly higher nitrogen fixers (42.7 and $39.8 \times 103 \mathrm{cfu} \mathrm{g}^{-1}$ soil), PSB (44.2 and $41.8 \times 104 \mathrm{cfu} \mathrm{g}$ soil), Azospirillum spp. population (3.95 and $0.80 \times 106$ cfu g- 1 soil), dehydrogenase (15.7 and $14.3 \mathrm{~g} \mathrm{TPF} \mathrm{g}$ ${ }^{1}$ soil day $\left.{ }^{-1}\right)$ and phosphatase ( 29.9 and $28.7 \mathrm{~g} \mathrm{pNP} \mathrm{g}^{-1}$ soil $^{-1}$ ) at flowering and harvest, respectively.

Interpretation : Higher soil microbial population viz., N fixers, PSB, Azospirillum spp. and soil enzyme activity of dehydrogenase and phosphatase at flowering and harvest was with the application of FYM (1/3) + vermicompost $(1 / 3)+$ green leaf manure (1/3) equivalent to RDN of $100 \mathrm{~kg}^{-1}$ with recommended FYM $\left(5\right.$ tha $\left.^{-1}\right)$ and microbial consortium (Azospirillum + PSB) with soil application of biodigester in aerobic rice.

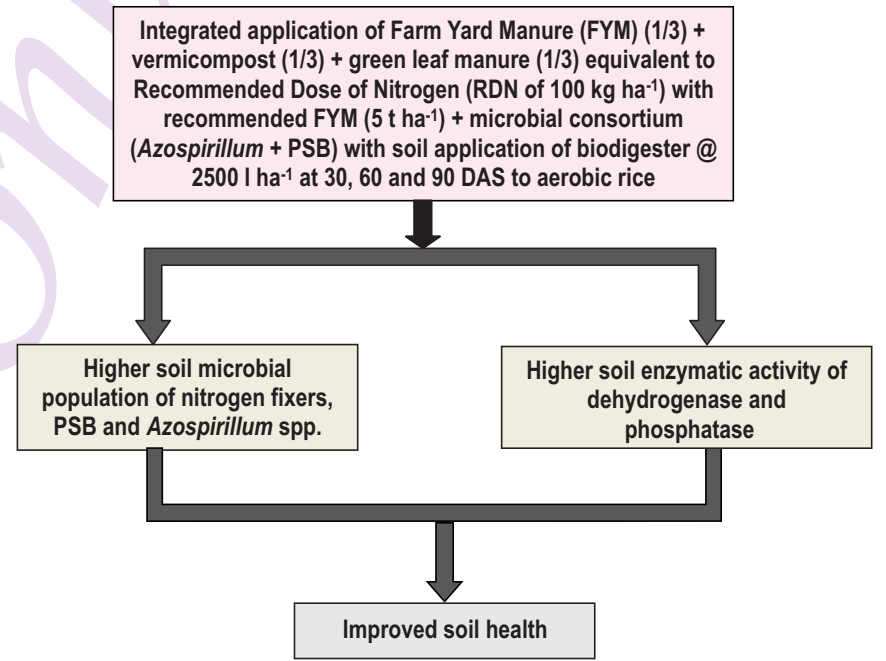




\section{Introduction}

The natural ability of soil to support optimum growth and yield of crops depends not only on its physical and chemical properties, but also on the intensity of biological processes. Soil microbes mediate the biochemical transformations of organic matter that underpin essential ecosystem functions, including decomposition, mineralization of plant available nutrients, and nutrient retention. Organic production relies on these microbiallyderived ecosystem functions and thus, may be a model system for ecological intensification of agriculture (Jackson et al., 2012). Soil fertility at great extent is controlled by different biochemical activities of the microflora, especially in the immediate surroundings of the roots, the rhizosphere, which under the influence of roots, carry a particularly dense population of microorganisms. Microorganisms can alter the degree of nutrient supply to higher plants through decomposition of organic compounds, immobilization of available nutrients, mineralization of nutrients or by promoting solubilization of fixed or insoluble mineral forms. The quantity and quality of soil organic matter and carbon and nitrogen inputs are the overriding controls on soil microbial biomass and activity. Thus, distinct organic amendments (e.g. manure, leguminous cover crops, and composted materials) can stimulate microbial biomass differently through increased labile organic matter (Kallenbach and Grandy, 2011). Soil enzymes are a group of enzymes present in soil and are continuously playing important role in maintaining soil ecology, physical and chemical properties, fertility and health. These enzymes also play a key role in biochemical functions in the overall process of organic matter decomposition in soil system. They are important in catalyzing several vital reactions necessary for the life processes of micro-organisms in soil and stabilization of soil structure, decomposition of organic wastes, organic matter formation and nutrient cycling and hence, play an important role in agriculture. The enzymatic activities of soil catalyzes the biochemical activities performed by bacteria and thereby, indicates the potential of soil to permit the basic biochemical processes necessary for maintaining soil fertility. Organic management increases overall enzyme activity (Moeskops et al., 2010), but activities of specific enzymes may change depending on the composition of the amendments and the relative availability of nutrients, as well as other factors, such as soil type and its unique characteristics (Stursova and Baldrian, 2010). Municipal wastewater sludge amendment apparently increased urease, dehydrogenase, alkaline phosphatase and glucosidase activities in soil by 48-70\%, 14-47\%, 33-66\% and 9$14 \%$, respectively (Efsun et al., 2015). Dehydrogenase activity estimates overall microbial activity due to its presence in all the microorganisms (Taylor et al., 2002). Phosphatase activity is related to phosphorus cycle in soil (Aon and Colaneri, 2001). Whereas, higher phosphatase activity increases significantly in typical purple soil irrigated by biogas slurry in China (Shiling et al., 2015). Therefore, while studying the effect of different levels and sources of fertilization on soil fertility, attention must be focused to the activity of the micro flora and enzymatic activity towards soil biological quality.

In aerobic rice (Oryza sativa) among the various practices, optimizing the use of manures and fertilizers is one of the important strategies for increasing productivity of rice. The contribution of organic manures is to be judged not only in terms of nutrient contribution, but also by their role in building up nutrient reserves in soil and increasing organic matter level of soil which ultimately improves physical, chemical and biological properties of soil. Using organic sources like farm yard manure, compost, vermicompost, sheep and goat manure and poultry manure in combination with liquid organic manures like cattle urine, panchgavya, vermiwash, bio-digested liquid, jeevamrut etc. deserves priority for sustained production and better on farm resource recycling and utilization. Liquid organic manure provides nutrients, growth promoting substances, as well as they are microbial enriched products that play a vital role in organic rice production. In the present investigation, an attempt was made to examine the influence of different levels and sources of fertilization on dynamics of soil microbial biomass and enzyme activities in vertisols under aerobic rice cultivation.

\section{Materials and Methods}

A field experiment was conducted during kharif 2013 and 2014 at Main Agricultural Research Station, UAS, Dharwad, Karnataka. The soil of the experimental site was clayey, having medium carbon $\left(4.9 \mathrm{~g} \mathrm{~kg}^{-1}\right)$ and available $\mathrm{N} \mathrm{P} \mathrm{K}$ (225.79:23.30:285.60 NPK kg ha-1). The experiment was laid out in a split plot design with three replications and comprised of six manurial combinations as main plots $\left(\mathrm{M}_{1}-\right.$ Recommended dose of chemical fertilizer (RDF) (100:50:50 kg N, $\mathrm{P}_{2} \mathrm{O}_{5}, \mathrm{~K}_{2} \mathrm{O} \mathrm{ha}^{-1}$ ); $\mathrm{M}_{2}$ - RDF +Farm yard manure ( 5 t ha $\left.^{-1}\right)+$ Microbial consortium (Azospirillum + Phosphate solubilizing bacteria) (MC); $\mathrm{M}_{3}-\mathrm{FYM}(1 / 3)$ + vermicompost $(1 / 3)+$ green leaf manure (1/3) equivalent to RDN; $M_{4}$ - FYM (1/3) + vermicompost (1/3) + green leaf manure (1/3) equivalent to RDN + microbial consortium; $M_{5}-F Y M(1 / 3)+$ vermicompost $(1 / 3)+$ green leaf manure (1/3) equivalent to RDN with recommended FYM ( 5 tha $\left.{ }^{-1}\right) ; M_{6}-F Y M(1 / 3)$ + vermicompost $(1 / 3)+$ green leaf manure (1/3) equivalent to RDN with recommended $\mathrm{FYM}+$ microbial consortium) and four liquid organic manure treatments as sub plots $\left(\mathrm{L}_{1}\right.$ : Soil application of jeevamrut @ $500 \mathrm{I} \mathrm{ha}^{-1}$ at 30,60 and $90 \mathrm{DAS}, \mathrm{L}_{2}$ : Foliar application of cow urine @ $500 \mathrm{I}$ ha ${ }^{-1}$ at 30,60 and 90 DAS, $\mathrm{L}_{3}$ : Soil application of biodigester @ 2500 Iha ${ }^{-1}$ at 30,60 and 90 DAS, L : Control-no liquid manure application). Organic manures equivalent to RDN through FYM, vermicompost and green leaf manure (Gliricidia spp.) were applied 15 days before sowing as per treatments. The chemical fertilizers as per the recommended package alone and along with FYM were applied. The seeds of variety MAS-26 
developed specifically for aerobic rice production from UAS, Bengaluru were used. Liquid organic manures were applied as per schedule to the respective plots. Neem oil, Metarrhizium and Pseudomonas fluroscence were sprayed to manage the insect pests and diseases in organic nutrient management treatments.

Determination of microbes : Rhizosphere soil was collected at flowering stage and harvested by uprooting plants from each plot and keeping the soil around root system intact for determination of soil biological properties. Microbial population in soil before sowing of crop and after harvest was determined by standard serial dilution plate count method. Ten grams of soil (treatment wise) was weighed and mixed in $90 \mathrm{ml}$ sterilized water blank to give $10^{1}$ dilutions. Subsequent dilutions up to $10^{4}$ were made by transferring serially $1 \mathrm{ml}$ of each dilution to $9 \mathrm{ml}$ of sterilized water blanks. The population of free living $\mathrm{N}$ fixture - Azotobacter, Phosphate solubilizing bacteria (PSB) were estimated by standard serial dilution plate count method and by taking $1 \mathrm{ml}$ from selected dilution of $10^{3}, 10^{4}$ were transferred aseptically to petridishes and the desired melted agar media ware added to the respective dilutions. Plating on appropriate media viz., soil extract agar for free living $\mathrm{N}$ fixture - Azotobacter and Pikovskaya's media for P-solubilizers was used. The plates were kept for incubation at $30^{\circ} \mathrm{C}+1^{\circ} \mathrm{C}$ for $24-48 \mathrm{hrs}$ and emerged colony forming units counted. To enumerate Azospirillum sp. in soil, the most probable number method was followed with semi solid Dobreiner medium. The tubes which turned blue and developed typical white subsurface pellicle were counted as positive or negative and were considered for the purpose of calculation by referring to statistical tables of Cochran (1950) and expressed as most probable number per gram of soil.

Enzyme assay : The dehydrogenase activity was determined by following the procedure described by Casida et al. (1964). Ten grams of soil and 0.2 grams of $\mathrm{CaCO}_{3}$ were thoroughly mixed in the conical flasks. Each flask was added with $3.0 \mathrm{ml}$ of $3 \% 2,3,5$ triphenyl tetrazolium chloride, $1.0 \mathrm{ml}$ of $1 \%$ glucose solution and $8.0 \mathrm{ml}$ of distilled water to leave a thin film of water above soil layer. At the end of incubation, the contents of the flask were rinsed down into small beaker and slurry was made by adding 10 $\mathrm{ml}$ of methanol. Repeated rinsing of soil with methanol was continued till the filtrate free of red colour. The filtrate was made upto $50 \mathrm{ml}$ with methanol in volumetric flask. The intensity of red colour was measured at $485 \mathrm{~nm}$ against blank using spectrometer. The Phosphatase activity in the soil samples was determined by following the procedure described by Evasi and Tabatabai (1979). The reaction mixture comprising of $1.0 \mathrm{~g}$ of soil, $0.2 \mathrm{ml}$ toluene, $4.0 \mathrm{ml}$ modified universal buffer $(\mathrm{pH} 7.5)$ and $1 \mathrm{ml}$ of $\mathrm{P}$ - nitro phenol phosphate solutions were mixed and incubated at $37^{\circ} \mathrm{C}$ for one hour. $1 \mathrm{ml}$ of $0.5 \mathrm{M} \mathrm{CaCl}_{2}$ and $4 \mathrm{ml}$ of $0.5 \mathrm{NaOH}$ were added, swirled and filtered. The intensity of yellow colour was measured at $420 \mathrm{~nm}$ against the reagent blank.

Statistical analysis : The experimental data obtained were subjected to statistical analysis by adopting Fisher's method of analysis of variance as outlined by Gomez and Gomez (1984). The level of significance used in ' $F$ ' test was $5 \%$. The mean values of main plot, sub plot and interaction were separately subjected to Duncan' multiple range test using the corresponding error mean sum of squares and degrees of freedom values. Values indicated with same alphabet did not differ significantly.

\section{Results and Discussion}

Microbial population : Application of FYM (1/3) + vermicompost $(1 / 3)+$ green leaf manure (1/3) equivalent to RDN with recommended FYM + microbial consortium $\left(\mathrm{M}_{6}\right)$ recorded significantly higher nitrogen fixers ( 40.7 and $37.4 \times 10^{3} \mathrm{cfu} \mathrm{g}^{-1}$ soil), PSB (42.6 and $40.3 \times 10^{4} \mathrm{cfu} \mathrm{g}^{-1}$ soil) and Azospirillum spp. population (2.94 and $0.51 \times 10^{6} \mathrm{cfu}^{-1}$ soil) at flowering and harvest respectively (Table 1 and 2). The rate of increase in the population of nitrogen fixers, PSB, Azospirillum spp. population during flowering decline subsequently till harvest corroborated with the growth and maturity of the crop. Iswandi et al. (2011) reported that rice root exudates contain some organic compounds as a source of nutrition for microorganisms and encourages colonization in the population of Azospirillum spp. and PSB during flowering stage of rice crop. Among the liquid organic manures, soil application of biodigester @ 2500 I ha $^{-1}$ at 30,60 and 90 DAS recorded significantly higher nitrogen fixers (34.4 and $31.6 \times 10^{3} \mathrm{cfu} \mathrm{g}^{-1}$ soil) PSB (31.0 and $28.3 \times 10^{4} \mathrm{cfu} \mathrm{g}^{-1}$ soil) and Azospirillum sp. population (1.90 and $0.31 \times 10^{6} \mathrm{cfu} \mathrm{g}^{-1}$ soil) at flowering and harvest respectively. Among the treatment combinations, integrated application of FYM (1/3) + vermicompost (1/3) + green leaf manure (1/3) equivalent to RDN with recommended FYM + microbial consortium with soil application of biodigester @ 2500 I ha ${ }^{-1}$ at 30, 60 and 90 DAS $\left(\mathrm{M}_{6} \mathrm{~L}_{3}\right)$ recorded significantly higher nitrogen fixers (42.7 and 39.8 $\mathrm{x} 10^{3} \mathrm{cfu} \mathrm{g}^{-1}$ soil), PSB (44.2 and $41.8 \times 10^{4} \mathrm{cfu}^{-1}$ soil) and Azospirillum spp. population (3.95 and $0.80 \times 10^{6} \mathrm{cfu} \mathrm{g}^{-1}$ soil) at flowering and harvest respectively. Ponmurugan and Gopi (2006) and Saini et al. (2005) reported the synergistic interactions between introduced microbial inoculants and the native microorganisms in the rhizosphere of crop. Use of liquid organic manures with organic manures was also helpful in improving the microbial population. These observations indicate that repeated use of liquid organic manures would help in enhanced bacterial activity and ultimately in improved soil fertility. Further continuous use of liquid organic manures with organic manures can improve microbial activity in soil and these improving the fertility in short run (Sudhanshu et al., 2009). Similarly, observation on positive role of sugar on microbial activity was documented by Buenaventurada and Tokiwa (2007). Majumdar et al. (2006) inferred that application of urine to the soil resulted in increased microflora of soil. Biodigester has helped in improving soil physical, chemical and biological properties of soil leading to 
Table 1: Effect of manurial practices and liquid organic manures on $\mathrm{N}$ fixures $\left(\mathrm{x} 10^{3} \mathrm{cfu} \mathrm{g}^{-1}\right.$ soil) and PSB $\left(\mathrm{x}^{1} 0^{4} \mathrm{cfu} \mathrm{g}^{-1}\right.$ soil) in rhizosphere

\begin{tabular}{|c|c|c|c|c|c|c|c|c|c|c|c|c|}
\hline \multirow[t]{3}{*}{ Treatments } & \multicolumn{6}{|c|}{ N fixures } & \multicolumn{6}{|c|}{ PSB } \\
\hline & \multicolumn{3}{|c|}{ Flowering } & \multicolumn{3}{|c|}{ Harvest } & \multicolumn{3}{|c|}{ Flowering } & \multicolumn{3}{|c|}{ Harvest } \\
\hline & 2013 & 2014 & Pooled & 2013 & 2014 & Pooled & 2013 & 2014 & Pooled & 2013 & 2014 & Pooled \\
\hline \multicolumn{13}{|l|}{ Manurial practices (M) } \\
\hline $\mathrm{M}_{1}-\mathrm{RDF}\left(100: 50: 50 \mathrm{~kg} \mathrm{~N}, \mathrm{P}_{2} \mathrm{O}_{5}, \mathrm{~K}_{2} \mathrm{Oha}^{-1}\right)$ & $21.0^{f}$ & $24.3^{f}$ & $22.6^{f}$ & $19.3^{f}$ & $21.6^{f}$ & $20.4^{\dagger}$ & $18.3^{f}$ & $19.0^{t}$ & $18.7^{f}$ & $14.7^{\mathrm{f}}$ & $15.3^{f}$ & $15.0^{f}$ \\
\hline$M_{2}-R D F+F Y M+M C$ & $25.0^{\mathrm{e}}$ & $28.4^{\mathrm{e}}$ & $26.7^{\mathrm{e}}$ & $23.4^{\mathrm{e}}$ & $25.8^{\mathrm{e}}$ & $24.6^{\mathrm{e}}$ & $28.7^{\circ}$ & $34.4^{\mathrm{c}}$ & $31.5^{\mathrm{c}}$ & $26.3^{\mathrm{c}}$ & $32.1^{\circ}$ & $29.2^{c}$ \\
\hline $\begin{array}{l}M_{3}-F Y M(1 / 3)+V C(1 / 3)+G L M(1 / 3) \\
\text { equi.RDN }\end{array}$ & $31.0^{d}$ & $34.0^{d}$ & $32.5^{\mathrm{d}}$ & $28.0^{\mathrm{d}}$ & $30.3^{d}$ & $29.2^{d}$ & $20.3^{\mathrm{e}}$ & $24.3^{e}$ & $22.3^{\mathrm{e}}$ & $17.7^{\mathrm{e}}$ & $21.7^{\circ}$ & $19.7^{e}$ \\
\hline $\begin{array}{l}\mathrm{M}_{4}-\mathrm{FYM}(1 / 3)+\mathrm{VC}(1 / 3)+\mathrm{GLM}(1 / 3) \\
\text { equi.RDN+MC }\end{array}$ & $33.0^{\circ}$ & $36.0^{\circ}$ & $34.5^{c}$ & $30.0^{\circ}$ & $32.3^{c}$ & $31.2^{c}$ & $32.7^{b}$ & $40.3^{b}$ & $36.5^{b}$ & $30.3^{b}$ & $38.0^{b}$ & $34.2^{b}$ \\
\hline $\begin{array}{l}\mathrm{M}_{5}-\mathrm{FYM}(1 / 3)+\mathrm{VC}(1 / 3)+\mathrm{GLM}(1 / 3) \\
\text { equi.RDN+FYM }\end{array}$ & $36.9^{b}$ & $40.0^{b}$ & $38.5^{b}$ & $34.0^{\mathrm{b}}$ & $36.3^{b}$ & $35.2^{b}$ & $22.3^{d}$ & $26.6^{d}$ & $24.4^{d}$ & $19.7^{d}$ & $23.9^{d}$ & $21.8^{d}$ \\
\hline $\begin{array}{l}M_{6}-F Y M(1 / 3)+V C(1 / 3)+G L M(1 / 3) \\
\text { qui.RDN+FYM+MC }\end{array}$ & $39.1^{a}$ & $42.3^{\mathrm{a}}$ & $40.7^{\mathrm{a}}$ & $36.3^{\mathrm{a}}$ & $38.6^{\mathrm{a}}$ & $37.4^{\mathrm{a}}$ & $38.4^{a}$ & $46.8^{\mathrm{a}}$ & $42.6^{a}$ & $36.1^{a}$ & $44.4^{\mathrm{a}}$ & $40.3^{a}$ \\
\hline $\begin{array}{l}\text { S.Em } \pm \\
\text { Liquid organic manures }(\mathrm{L})\end{array}$ & 0.27 & 0.21 & 0.16 & 0.21 & 0.38 & 0.29 & 0.25 & 0.36 & 0.26 & 0.49 & 0.37 & 0.38 \\
\hline $\begin{array}{l}\text { L,-Jeevamrut @ } 500 \text { litres ha }^{-1} \text { at } 30, \\
60 \text { and } 90 \text { DAS }\end{array}$ & $31.4^{b}$ & $34.8^{b}$ & $33.1^{\mathrm{b}}$ & $29.1^{b}$ & $31.4^{b}$ & $30.3^{b}$ & $27.2^{b}$ & $32.3^{b}$ & $29.8^{b}$ & $24.6^{b}$ & $29.7^{\circ}$ & $27.1^{b}$ \\
\hline $\begin{array}{l}\mathrm{L}_{2}-\text { Cow urine @ } 500 \text { litres ha }{ }^{-1} \text { at } 30, \\
60 \text { and } 90 \text { DAS }\end{array}$ & $30.6^{c}$ & $33.4^{\circ}$ & $32.0^{\circ}$ & $27.7^{\circ}$ & $30.1^{\circ}$ & $28.9^{\circ}$ & $26.4^{\circ}$ & $31.4^{\circ}$ & $28.9^{\circ}$ & $23.7^{\circ}$ & $28.8^{c}$ & $26.2^{\circ}$ \\
\hline $\begin{array}{l}\mathrm{L}_{3}-\text { Bio digester @ } 2500 \text { litres ha }{ }^{-1} \text { at } 30 \text {, } \\
60 \text { and } 90 \text { DAS }\end{array}$ & $32.7^{\mathrm{a}}$ & $36.1^{\mathrm{a}}$ & $34.4^{\mathrm{a}}$ & $30.4^{a}$ & $32.8^{\mathrm{a}}$ & $31.6^{\mathrm{a}}$ & $28.4^{a}$ & $33.6^{\mathrm{a}}$ & $31.0^{\mathrm{a}}$ & $25.8^{\mathrm{a}}$ & $30.8^{\mathrm{a}}$ & $28.3^{\mathrm{a}}$ \\
\hline $\mathrm{L}_{4}$-Control (no liquid manure application) & $29.3^{d}$ & $32.3^{\mathrm{d}}$ & $30.8^{d}$ & $26.7^{d}$ & $29.0^{d}$ & $27.8^{\mathrm{d}}$ & $25.1^{\mathrm{d}}$ & $30.2^{d}$ & $27.7^{\mathrm{d}}$ & $22.5^{\mathrm{d}}$ & $27.6^{d}$ & $25.1^{\mathrm{d}}$ \\
\hline $\begin{array}{l}\text { S.Em } \pm \\
\text { Interactions-(MXL) }\end{array}$ & 0.18 & 0.19 & 0.12 & 0.19 & 0.24 & 0.20 & 0.18 & 0.18 & 0.18 & 0.18 & 0.18 & 0.18 \\
\hline$M_{1} L_{1}$ & $21.3^{n}$ & $24.7^{\mathrm{n}}$ & $23.0^{\circ}$ & $19.7^{\circ}$ & $22.0^{\mathrm{mn}}$ & $20.8^{\circ}$ & $18.7^{\mathrm{mn}}$ & $19.3^{\circ}$ & $19.0^{\circ}$ & $15.0^{\text {no }}$ & $15.7^{\circ}$ & $15.3^{p}$ \\
\hline$M_{1} L_{2}$ & $20.7^{n}$ & $23.3^{\text {no }}$ & $22.0^{p}$ & $18.3^{\text {op }}$ & $20.7^{\text {no }}$ & $19.5^{\circ p}$ & $18.0^{n}$ & $18.7^{\circ}$ & $18.3^{\circ}$ & $14.3^{\circ}$ & $15.0^{\circ}$ & $14.7^{p}$ \\
\hline$M_{1} L_{3}$ & $22.7^{\mathrm{m}}$ & $26.7^{\mathrm{m}}$ & $24.7^{\mathrm{n}}$ & $21.7^{n}$ & $24.0^{\prime}$ & $22.8^{n}$ & $20.0^{\mathrm{lm}}$ & $20.7^{\mathrm{n}}$ & $20.3^{n}$ & $16.3^{\mathrm{mn}}$ & $17.0^{\mathrm{n}}$ & $16.7^{\circ}$ \\
\hline$M_{1} L_{4}$ & $19.3^{\circ}$ & $22.3^{\circ}$ & $20.8^{9}$ & $17.3^{p}$ & $19.7^{\circ}$ & $18.5^{p}$ & $16.7^{\circ}$ & $17.3^{p}$ & $17.0^{\circ}$ & $13.0^{p}$ & $13.7^{p}$ & $13.3^{9}$ \\
\hline$M_{2} L_{1}$ & $25.7^{k}$ & $29.3^{1}$ & $27.5^{1}$ & $24.3^{\mathrm{m}}$ & $26.7^{k}$ & $25.5^{\mathrm{m}}$ & $29.3^{9}$ & $35.3^{9}$ & $32.3^{9}$ & $2700^{\circ}$ & $33.0^{9}$ & $30.0^{9}$ \\
\hline $\mathrm{M}_{2} \mathrm{~L}_{2}$ & $24.3^{\prime}$ & $27.3^{\mathrm{m}}$ & $25.8^{\mathrm{m}}$ & $22.3^{n}$ & $24.7^{1}$ & $23.5^{\mathrm{n}}$ & $28.0^{\mathrm{h}}$ & $33.7^{\mathrm{h}}$ & $30.8^{h}$ & $25.7^{\mathrm{h}}$ & $31.3^{\mathrm{h}}$ & $28.5^{\mathrm{h}}$ \\
\hline $\mathrm{M}_{2} \mathrm{~L}_{3}$ & $26.7^{k}$ & $30.7^{1}$ & $28.7^{\mathrm{k}}$ & $25.7^{\mathrm{m}}$ & $28.0^{\mathrm{jk}}$ & $26.8^{\operatorname{lm}}$ & $30.3^{f g}$ & $36.0^{9}$ & $33.2^{9}$ & $28.0^{f g}$ & $33.7^{9}$ & $30.8^{9}$ \\
\hline $\mathrm{M}_{2} \mathrm{~L}_{4}$ & $23.3^{\mathrm{Im}}$ & $26.3^{\mathrm{m}}$ & $24.8^{n}$ & $21.3^{n}$ & $23.7^{\mathrm{mm}}$ & $22.5^{\mathrm{n}}$ & $27.0^{\mathrm{h}}$ & $32.7^{\mathrm{h}}$ & $29.8^{h}$ & $24.7^{\mathrm{h}}$ & $30.3^{\mathrm{h}}$ & $27.5^{\mathrm{h}}$ \\
\hline $\mathrm{M}_{3} \mathrm{~L}_{1}$ & $31.3^{\mathrm{hi}}$ & $34.7^{\mathrm{j}}$ & $33.0^{\mathrm{h}}$ & $28.7^{\mathrm{j}}$ & $31.0^{\mathrm{hi}}$ & $29.8^{\mathrm{ij}}$ & $20.7^{k 1}$ & $24.3^{\prime}$ & $22.5^{\mathrm{lm}}$ & $18.0^{k /}$ & $21.7^{\mathrm{lm}}$ & $19.8^{\mathrm{lm}}$ \\
\hline $\mathrm{M}_{3} \mathrm{~L}_{2}$ & $30.7^{i}$ & $33.3^{\mathrm{ik}}$ & $32.0^{\prime}$ & $27.3^{j k}$ & $29.7^{\mathrm{j}}$ & $28.5^{\mathrm{jk}}$ & $20.0^{\mathrm{lm}}$ & $24.0^{\prime}$ & $22.0^{\mathrm{m}}$ & $17.3^{\mathrm{lm}}$ & $21.3^{\operatorname{lm}}$ & $19.3^{\mathrm{mn}}$ \\
\hline$M_{3} L_{3}$ & $32.7^{g h}$ & $35.7^{\mathrm{hi}}$ & $34.2^{9}$ & $29.7^{\mathrm{hi}}$ & $32.0^{\mathrm{gh}}$ & $30.8^{\mathrm{hi}}$ & $22.0^{\mathrm{ik}}$ & $26.0^{\mathrm{ik}}$ & $24.0^{\mathrm{jk}}$ & $19.3^{\mathrm{jk}}$ & $23.3^{\mathrm{j}}$ & $21.3^{\mathrm{jk}}$ \\
\hline $\mathrm{M}_{3} \mathrm{~L}_{4}$ & $29.3^{j}$ & $32.3^{k}$ & $30.8^{j}$ & $26.3^{\mathrm{kl}}$ & $28.7^{j}$ & $27.5^{\mathrm{kl}}$ & $18.7^{\mathrm{mn}}$ & $22.7^{\mathrm{m}}$ & $20.7^{\mathrm{n}}$ & $16.0^{\mathrm{mn}}$ & $20.3^{\mathrm{m}}$ & $18.2^{n}$ \\
\hline$M_{4} L_{1}$ & $33.3^{9}$ & $36.7^{\text {gh }}$ & $35.0^{9}$ & $30.7^{\text {gh }}$ & $33.0^{f g}$ & $31.8^{\text {gh }}$ & $33.0^{\mathrm{e}}$ & $40.7^{\circ}$ & $36.8^{\circ}$ & $30.7^{e}$ & $38.3^{\mathrm{e}}$ & $34.5^{\mathrm{e}}$ \\
\hline $\mathrm{M}_{4} \mathrm{~L}_{2}$ & $32.7^{\text {gh }}$ & $35.7^{\mathrm{hi}}$ & $34.2^{9}$ & $29.7^{\text {hi }}$ & $32.0^{\mathrm{gh}}$ & $30.8^{\text {hi }}$ & $32.3^{\mathrm{e}}$ & $40.0^{\circ}$ & $36.2^{\mathrm{e}}$ & $30.0^{\mathrm{e}}$ & $37.7^{\mathrm{e}}$ & $33.8^{e}$ \\
\hline $\mathrm{M}_{4} \mathrm{~L}_{3}$ & $34.7^{\prime}$ & $37.3^{\text {tg }}$ & $36.0^{\dagger}$ & $31.3^{\text {tg }}$ & $33.7^{\mathrm{fg}}$ & $32.5^{f g}$ & $34.3^{\mathrm{d}}$ & $42.0^{d}$ & $38.2^{d}$ & $32.0^{d}$ & $39.7^{d}$ & $35.8^{d}$ \\
\hline $\mathrm{M}_{4} \mathrm{~L}_{4}$ & $31.3^{\text {hi }}$ & $34.3^{\mathrm{j}}$ & $32.8^{\mathrm{hi}}$ & $28.3^{i j}$ & $30.7^{\mathrm{hi}}$ & $29.5^{\mathrm{j}}$ & $31.0^{\circ}$ & $38.7^{\dagger}$ & $34.8^{f}$ & $28.7^{t}$ & $36.3^{f}$ & $32.5^{f}$ \\
\hline$M_{5} L_{1}^{4}$ & $37.3^{\mathrm{cd}}$ & $40.7^{\text {cd }}$ & $39.0^{d}$ & $34.7^{\text {cd }}$ & $37.0^{\text {cd }}$ & $35.8^{\mathrm{cd}}$ & $22.7^{\mathrm{j}}$ & $27.0^{j}$ & $24.8^{\mathrm{j}}$ & $20.0^{j}$ & $24.3^{\mathrm{j}}$ & $22.2^{j}$ \\
\hline$M_{5} L_{2}$ & $36.3^{\text {de }}$ & $39.3^{\text {de }}$ & $37.8^{\mathrm{e}}$ & $33.3^{\text {de }}$ & $35.7^{\mathrm{de}}$ & $34.5^{\mathrm{de}}$ & $21.7^{\mathrm{jk}}$ & $26.0^{\mathrm{ik}}$ & $23.8^{j-1}$ & $19.0^{\mathrm{ik}}$ & $23.3^{\mathrm{k}}$ & $21.2^{j-1}$ \\
\hline$M_{5} L_{3}$ & $38.7^{b c}$ & $41.7^{\mathrm{bc}}$ & $40.2^{c}$ & $35.7^{\mathrm{bc}}$ & $38.0^{\mathrm{bc}}$ & $36.8^{\mathrm{bc}}$ & $24.0^{\prime}$ & $28.3^{i}$ & $26.2^{i}$ & $21.3^{i}$ & $25.7^{i}$ & $23.5^{i}$ \\
\hline$M_{5} L_{4}$ & $35.3^{\text {ef }}$ & $38.3^{\text {ef }}$ & $36.8^{f}$ & $32.3^{\text {ef }}$ & $34.7^{\text {ef }}$ & $33.5^{\text {ef }}$ & $20.7^{k 1}$ & $25.0^{k 1}$ & $22.8^{k-m}$ & $18.3^{\mathrm{kl}}$ & $22.3^{k 1}$ & $20.3^{k-m}$ \\
\hline$M_{6} L_{1}$ & $39.7^{\mathrm{ab}}$ & $42.7^{b}$ & $41.2^{b}$ & $36.7^{b}$ & $39.0^{b}$ & $37.8^{\mathrm{b}}$ & $39.0^{\mathrm{ab}}$ & $47.3^{\mathrm{ab}}$ & $43.2^{\mathrm{ab}}$ & $36.7^{\mathrm{ab}}$ & $45.0^{\mathrm{ab}}$ & $40.8^{\mathrm{ab}}$ \\
\hline$M_{6} L_{2}$ & $38.7^{\mathrm{bc}}$ & $41.3^{\text {bc }}$ & $40.0^{\circ}$ & $35.3^{\mathrm{bc}}$ & $37.7^{\mathrm{bc}}$ & $36.5^{\mathrm{bc}}$ & $38.0^{\mathrm{b}}$ & $46.3^{b}$ & $42.2^{b}$ & $35.7^{\mathrm{b}}$ & $44.0^{b}$ & $39.8^{\mathrm{b}}$ \\
\hline$M_{6} L_{3}$ & $40.7^{a}$ & $44.7^{\mathrm{a}}$ & $42.7^{\mathrm{a}}$ & $38.7^{\mathrm{a}}$ & $41.0^{\mathrm{a}}$ & $39.8^{\mathrm{a}}$ & $40.0^{\mathrm{a}}$ & $48.3^{\mathrm{a}}$ & $44.2^{\mathrm{a}}$ & $37.7^{\mathrm{a}}$ & $46.0^{\mathrm{a}}$ & $41.8^{\mathrm{a}}$ \\
\hline$M_{6} L_{4}$ & $37.3^{\mathrm{cd}}$ & $40.3^{\text {cd }}$ & $38.8^{d}$ & $34.3^{\text {cd }}$ & $36.7^{\text {cd }}$ & $35.5^{\text {cd }}$ & $36.7^{\circ}$ & $45.0^{\circ}$ & $40.8^{\circ}$ & $34.3^{c}$ & $42.7^{\circ}$ & $38.5^{\mathrm{c}}$ \\
\hline S.Em \pm & 0.45 & 0.46 & 0.31 & 0.48 & 0.59 & 0.51 & 0.45 & 0.44 & 0.44 & 0.45 & 0.44 & 0.45 \\
\hline Initial & \multicolumn{6}{|c|}{16.67} & \multicolumn{6}{|c|}{12.67} \\
\hline
\end{tabular}

Means followed by the same alphabet do not differ significantly by DMRT $(0.05)$ 
Table 2 : Effect of manurial practices and liquid organic manures on Azospirillum spp. ( $\times 10^{6} \mathrm{cfu} \mathrm{g}^{-1}$ soil) in rhizosphere

\begin{tabular}{|c|c|c|c|c|c|c|}
\hline \multirow[t]{2}{*}{ Treatments } & \multicolumn{3}{|c|}{ Flowering } & \multicolumn{3}{|c|}{ Harvest } \\
\hline & 2013 & 2014 & Pooled & 2013 & 2014 & Pooled \\
\hline \multicolumn{7}{|l|}{ Manurial practices (M) } \\
\hline $\mathrm{M}_{1}-\mathrm{RDF}\left(100: 50: 50 \mathrm{~kg} \mathrm{~N}, \mathrm{P}_{2} \mathrm{O}_{5} \mathrm{~K}_{2} \mathrm{O} \mathrm{ha}^{-1}\right)$ & $0.08^{\circ}$ & $0.10^{\circ}$ & $0.09^{\circ}$ & $0.02^{d}$ & $0.03^{d}$ & $0.03^{\mathrm{d}}$ \\
\hline$M_{2}-R D F+F Y M+M C$ & $1.56^{\mathrm{b}}$ & $1.75^{b}$ & $1.65^{\mathrm{b}}$ & $0.17^{\circ}$ & $0.20^{\mathrm{c}}$ & $0.19^{c}$ \\
\hline$M_{3}-F Y M(1 / 3)+V C(1 / 3)+G L M(1 / 3)$ equi. RDN & $0.31^{\mathrm{c}}$ & $0.43^{c}$ & $0.37^{\circ}$ & $0.05^{d}$ & $0.06^{d}$ & $0.05^{\mathrm{d}}$ \\
\hline$M_{4}-F Y M(1 / 3)+V C(1 / 3)+G L M(1 / 3)$ equi. $R D N+M C$ & $2.30^{\mathrm{a}}$ & $2.76^{\mathrm{a}}$ & $2.53^{\mathrm{a}}$ & $0.31^{b}$ & $0.43^{b}$ & $0.37^{b}$ \\
\hline$M_{5}-F Y M(1 / 3)+V C(1 / 3)+G L M(1 / 3)$ equi. RDN+FYM & $0.43^{\mathrm{c}}$ & $0.58^{\mathrm{c}}$ & $0.51^{\mathrm{c}}$ & $0.07^{d}$ & $0.09^{\text {cd }}$ & $0.08^{d}$ \\
\hline$M_{6}-F Y M(1 / 3)+V C(1 / 3)+G L M(1 / 3)$ equi. RDN+FYM+MC & $2.76^{\mathrm{a}}$ & $3.12^{\mathrm{a}}$ & $2.94^{\mathrm{a}}$ & $0.43^{\mathrm{a}}$ & $0.58^{\mathrm{a}}$ & $0.51^{\mathrm{a}}$ \\
\hline S.Em地 & 0.17 & 0.17 & 0.17 & 0.02 & 0.03 & 0.03 \\
\hline \multicolumn{7}{|l|}{ Liquid organic manures (L) } \\
\hline$L_{1}-$ Jeevamrut @ 500 litres ha ${ }^{-1}$ at 30,60 and 90 DAS & $1.50^{\mathrm{b}}$ & $1.75^{b}$ & $1.62^{b}$ & $0.19^{b}$ & $0.27^{b}$ & $0.23^{b}$ \\
\hline $\mathrm{L}_{2}$-Cow urine @ 500 litres ha $^{-1}$ at 30,60 and 90 DAS & $1.01^{\mathrm{c}}$ & $1.25^{\mathrm{c}}$ & $1.13^{\mathrm{c}}$ & $0.15^{\mathrm{c}}$ & $0.19^{c}$ & $0.17^{\circ}$ \\
\hline $\mathrm{L}_{3}$-Bio digester @ 2500 litres ha ${ }^{-1}$ at 30,60 and 90 DAS & $1.79^{a}$ & $2.02^{\mathrm{a}}$ & $1.90^{\mathrm{a}}$ & $0.27^{\mathrm{a}}$ & $0.35^{\mathrm{a}}$ & $0.31^{\mathrm{a}}$ \\
\hline $\mathrm{L}_{4}$-Control (no liquid manure application) & $0.66^{d}$ & $0.81^{d}$ & $0.74^{\mathrm{d}}$ & $0.10^{\mathrm{d}}$ & $0.13^{d}$ & $0.11^{\mathrm{d}}$ \\
\hline S.Em \pm & 0.05 & 0.06 & 0.05 & 0.01 & 0.01 & 0.01 \\
\hline \multicolumn{7}{|l|}{ Interactions-(M XL) } \\
\hline$M_{1} L_{1}$ & $0.10^{i}$ & $0.11^{k}$ & $0.11^{\prime}$ & $0.02^{j}$ & $0.03^{k}$ & $0.03^{\mathrm{jk}}$ \\
\hline$M_{1} L_{2}$ & $0.06^{i}$ & $0.08^{k}$ & $0.07^{\prime}$ & $0.02^{i}$ & $0.03^{k}$ & $0.02^{k}$ \\
\hline$M_{1} L_{3}$ & $0.12^{i}$ & $0.15^{k}$ & 0.14 & $0.04^{j}$ & $0.05^{\mathrm{jk}}$ & $0.04^{j k}$ \\
\hline$M_{1} L_{4}$ & $0.04^{i}$ & $0.06^{k}$ & $0.05^{\prime}$ & $0.01^{j}$ & $0.02^{k}$ & $0.02^{k}$ \\
\hline$M_{2} L_{1}$ & $1.73^{\mathrm{de}}$ & $1.87^{\mathrm{ef}}$ & $1.80^{\text {ef }}$ & $0.19^{d-f}$ & $0.22^{\mathrm{e}-9}$ & $0.21^{\text {ef }}$ \\
\hline $\mathrm{M}_{2} \mathrm{~L}_{2}$ & $1.43^{\text {ef }}$ & $1.53^{\mathrm{fg}}$ & $1.48^{\mathrm{fg}}$ & $0.15^{\mathrm{e}-\mathrm{h}}$ & $0.17^{\text {fih }}$ & $0.16^{\mathrm{e}-\mathrm{h}}$ \\
\hline $\mathrm{M}_{2} \mathrm{~L}_{3}$ & $2.13^{\text {cd }}$ & $2.47^{\text {cd }}$ & $2.30^{\mathrm{cd}}$ & $0.22^{\text {de }}$ & $0.26^{\mathrm{de}}$ & $0.24^{\mathrm{de}}$ \\
\hline $\mathrm{M}_{2} \mathrm{~L}_{4}$ & $0.95^{9}$ & $1.12^{\text {gh }}$ & $1.03^{\mathrm{hi}}$ & $0.13^{f-1}$ & $0.15^{g-i}$ & $0.14^{f-i}$ \\
\hline $\mathrm{M}_{3} \mathrm{~L}_{1}$ & $0.32^{\text {hi }}$ & $0.48^{i-k}$ & $0.40^{\mathrm{j}-1}$ & $0.05^{i j}$ & $0.07^{i-k}$ & $0.06^{i-k}$ \\
\hline $\mathrm{M}_{3} \mathrm{~L}_{2}$ & $0.27^{\mathrm{hi}}$ & $0.32^{\mathrm{jk}}$ & $0.30^{\mathrm{k}}$ & $0.04^{j}$ & $0.05^{\mathrm{jk}}$ & $0.05^{\mathrm{jk}}$ \\
\hline$M_{3} \mathrm{~L}_{3}$ & $0.48^{\mathrm{hi}}$ & $0.71^{\text {h.j }}$ & $0.60^{\mathrm{jk}}$ & $0.06^{i j}$ & $0.08^{i-k}$ & $0.07^{i-k}$ \\
\hline $\mathrm{M}_{3} \mathrm{~L}_{4}$ & $0.17^{i}$ & $0.21^{\mathrm{jk}}$ & $0.19^{k l}$ & $0.03^{j}$ & $0.04^{\mathrm{jk}}$ & $0.04^{\mathrm{jk}}$ \\
\hline$M_{4} L_{1}$ & $2.83^{b}$ & $3.53^{b}$ & $3.18^{b}$ & $0.32^{\circ}$ & $0.48^{\mathrm{C}}$ & $0.40^{\circ}$ \\
\hline $\mathrm{M} \mathrm{L}_{2}$ & $1.73^{\mathrm{de}}$ & $2.23^{\mathrm{de}}$ & $1.98^{\mathrm{de}}$ & $0.27^{\mathrm{cd}}$ & $0.32^{d}$ & $0.30^{\mathrm{d}}$ \\
\hline$M_{4} L_{3}$ & $3.53^{\mathrm{a}}$ & $3.77^{\mathrm{ab}}$ & $3.65^{\mathrm{a}}$ & $0.48^{b}$ & $0.71^{b}$ & $0.60^{b}$ \\
\hline $\mathrm{M}_{4} \mathrm{~L}_{4}$ & $1.10^{f g}$ & $1.50^{f g}$ & $1.30^{g h}$ & $0.17^{e-g}$ & $0.21^{e-g}$ & $0.19^{e-g}$ \\
\hline$M_{5} L_{1}$ & $0.48^{\mathrm{hi}}$ & $0.71^{\text {h-j }}$ & $0.60^{\mathrm{jk}}$ & $0.08^{h-j}$ & $0.10^{n-k}$ & $0.09^{h-k}$ \\
\hline $\mathrm{M}_{5} \mathrm{~L}_{2}$ & $0.32^{\mathrm{hi}}$ & $0.48^{i-k}$ & $0.40^{j-1}$ & $0.07^{\text {h-j }}$ & $0.08^{i-k}$ & $0.08^{n-k}$ \\
\hline $\mathrm{M}_{5} \mathrm{~L}_{3}$ & $0.71^{g h}$ & $0.88^{\mathrm{hi}}$ & $0.80^{i j}$ & $0.09^{9-j}$ & $0.12^{h-j}$ & $0.11^{g-j}$ \\
\hline $\mathrm{M}_{5} \mathrm{~L}_{4}$ & $0.21^{i}$ & $0.25^{\mathrm{jk}}$ & $0.23^{\mathrm{kl}}$ & $0.05^{\mathrm{ij}}$ & $0.07^{i-k}$ & $0.06^{i-k}$ \\
\hline$M_{6} L_{1}$ & $3.53^{\mathrm{a}}$ & $3.77^{a b}$ & $3.65^{\mathrm{a}}$ & $0.48^{b}$ & $0.71^{b}$ & $0.60^{b}$ \\
\hline $\mathrm{M}_{6} \mathrm{~L}_{2}$ & $2.23^{c}$ & $2.83^{c}$ & $2.53^{\circ}$ & $0.32^{c}$ & $0.48^{\circ}$ & $0.40^{\circ}$ \\
\hline $\mathrm{M}_{6} \mathrm{~L}_{3}$ & $3.77^{\mathrm{a}}$ & $4.13^{\mathrm{a}}$ & $3.95^{\mathrm{a}}$ & $0.71^{a}$ & $0.88^{\mathrm{a}}$ & $0.80^{\mathrm{a}}$ \\
\hline $\mathrm{M}_{6} \mathrm{~L}_{4}$ & $1.50^{\mathrm{ef}}$ & $1.73^{f}$ & $1.62^{e-g}$ & $0.21^{d-f}$ & $0.25^{\mathrm{d}-f}$ & $0.23^{\mathrm{de}}$ \\
\hline S.Em \pm & 0.14 & 0.15 & 0.12 & 0.02 & 0.02 & 0.02 \\
\hline Initial & & & & & & \\
\hline
\end{tabular}

Means followed by the same alphabet do not differ significantly by DMRT (0.05)

overall improvement in soil health in the long run (Mondal et al., 2003). Jeevamrutha was able to improve the microbial population when supplied on $\mathrm{N}$ basis.

Enzymatic activity : Application of FYM (1/3) + vermicompost $(1 / 3)+$ green leaf manure (1/3) equivalent to RDN with recommended $F Y M+$ microbial consortium $\left(M_{6}\right)$ recorded significantly higher dehydrogenase (14.6 and $13.1 \mathrm{~g} \mathrm{TPF} \mathrm{g}^{-1}$ soil day $\left.^{-1}\right)$ and phosphatase activity ( 28.5 and $27.3 \mathrm{~g} \mathrm{pNP} \mathrm{g}^{-1}$ soil $\left.^{-1}\right)$ at flowering and harvest respectively (Table 3). Among the liquid organic manures, soil application of biodigester @ 2500 I ha $^{-1}$ at 30, 60 and 90 DAS recorded significantly higher dehydrogenase (13.8 and $12.4 \mathrm{~g} \mathrm{TPF} \mathrm{g}^{-1}$ soil day ${ }^{-1}$ ) and phosphatase activity (27.0 and $25.7 \mathrm{~g} \mathrm{pNP} \mathrm{g}^{-1}$ soil $^{-1}$ ) at flowering and harvest respectively. Among the treatment combinations, integrated application of FYM (1/3) + vermicompost $(1 / 3)+$ green leaf manure (1/3) equivalent to RDN with recommended FYM + microbial consortium with soil application of biodigester @ 2500 I ha $^{-1}$ at 30 , 
Table 3 : Effect of manurial practices and liquid organic manures on dehyadrogenase ( $\mu \mathrm{g} \operatorname{TPF~g}^{-1}$ soil day $\left.^{-1}\right)$ and phosphatase $\left(\mu \mathrm{g} \mathrm{pNP} \mathrm{g}^{-1}\right.$ soil $\left.\mathrm{h}^{-1}\right)$ enzymes

\begin{tabular}{|c|c|c|c|c|c|c|c|c|c|c|c|c|}
\hline \multirow[t]{3}{*}{ Treatments } & \multicolumn{6}{|c|}{ Dehyadrogenase } & \multicolumn{6}{|c|}{ Phosphatase } \\
\hline & \multicolumn{3}{|c|}{ Flowering } & \multicolumn{3}{|c|}{ Harvest } & \multicolumn{3}{|c|}{ Flowering } & \multicolumn{3}{|c|}{ Harvest } \\
\hline & 2013 & 2014 & Pooled & 2013 & 2014 & Pooled & 2013 & 2014 & Pooled & 2013 & 2014 & Pooled \\
\hline \multicolumn{13}{|l|}{ Manurial practices (M) } \\
\hline $\mathrm{M}_{1}-\mathrm{RDF}\left(100: 50: 50 \mathrm{~kg} \mathrm{~N}, \mathrm{P}_{2} \mathrm{O}_{5}, \mathrm{~K}_{2} \mathrm{O} \mathrm{ha}^{-1}\right)$ & $10.1^{d}$ & $12.0^{d}$ & $11.0^{\circ}$ & $8.5^{\mathrm{d}}$ & $10.5^{\mathrm{e}}$ & $9.5^{\mathrm{d}}$ & $21.0^{d}$ & $23.0^{\circ}$ & $22.0^{\mathrm{d}}$ & $19.9^{d}$ & $21.8^{\mathrm{e}}$ & $20.9^{d}$ \\
\hline$M_{2}-R D F+F Y M+M C$ & $11.0^{\circ}$ & $12.8^{\circ}$ & $11.9^{d}$ & $9.5^{\mathrm{d}}$ & $11.4^{\mathrm{d}}$ & $10.4^{\circ}$ & $23.0^{c}$ & $24.8^{\mathrm{d}}$ & $23.9^{\circ}$ & $21.7^{\circ}$ & $23.4^{d}$ & $22.5^{\circ}$ \\
\hline $\begin{array}{l}\mathrm{M}_{3}-\mathrm{FYM}(1 / 3)+\mathrm{VC}(1 / 3)+\mathrm{GLM}(1 / 3) \\
\text { equi.RDN }\end{array}$ & $12.1^{b}$ & $14.0^{\mathrm{b}}$ & $13.0^{\circ}$ & $10.6^{c}$ & $12.5^{\circ}$ & $11.5^{\mathrm{b}}$ & $24.6^{\mathrm{b}}$ & $26.6^{\circ}$ & $25.6^{b}$ & $23.5^{b}$ & $25.4^{\mathrm{c}}$ & $24.4^{b}$ \\
\hline $\begin{array}{l}\mathrm{M}_{4}-\mathrm{FYM}(1 / 3)+\mathrm{VC}(1 / 3)+\mathrm{GLM}(1 / 3) \\
\text { equi.RDN+MC }\end{array}$ & $12.4^{b}$ & $14.3^{b}$ & $13.3^{c}$ & $10.9^{b c}$ & $12.8^{\circ}$ & $11.8^{b}$ & $25.0^{\mathrm{b}}$ & $27.0^{\circ}$ & $26.0^{b}$ & $23.9^{b}$ & $25.6^{c}$ & $24.8^{b}$ \\
\hline $\begin{array}{l}M_{5}-\mathrm{FYM}(1 / 3)+V C(1 / 3)+\mathrm{GLM}(1 / 3) \\
\text { equi.RDN+FYM }\end{array}$ & $13.3^{\mathrm{a}}$ & $15.1^{\mathrm{a}}$ & $14.2^{b}$ & $11.8^{\mathrm{ab}}$ & $13.6^{b}$ & $12.7^{\mathrm{a}}$ & $27.3^{\mathrm{a}}$ & $29.0^{\mathrm{b}}$ & $28.1^{a}$ & $26.1^{\mathrm{a}}$ & $27.7^{b}$ & $26.9^{a}$ \\
\hline $\begin{array}{l}M_{6}-F Y M(1 / 3)+V C(1 / 3)+G L M(1 / 3) \\
\text { qui.RDN+FYM+MC }\end{array}$ & $13.7^{\mathrm{a}}$ & $15.6^{\mathrm{a}}$ & $14.6^{\mathrm{a}}$ & $12.2^{\mathrm{a}}$ & $14.1^{\mathrm{a}}$ & $13.1^{\mathrm{a}}$ & $27.5^{\mathrm{a}}$ & $29.5^{\mathrm{a}}$ & $28.5^{\mathrm{a}}$ & $26.4^{\mathrm{a}}$ & $28.2^{\mathrm{a}}$ & $27.3^{\mathrm{a}}$ \\
\hline $\begin{array}{l}\text { S.Em } \pm \\
\text { Liquid organic manures (L) }\end{array}$ & 0.18 & 0.25 & 0.1 & 0.35 & 0.11 & 0.19 & 0.38 & 0.15 & 0.21 & 0.22 & 0.14 & 0.14 \\
\hline $\begin{array}{l}\text { L,-Jeevamrut @ } 500 \text { litres ha }^{-1} \text { at } 30, \\
60 \text { and } 90 \text { DAS }\end{array}$ & $12.4^{b}$ & $14.3^{\mathrm{a}}$ & $13.3^{b}$ & $10.9^{b}$ & $12.8^{b}$ & $11.8^{b}$ & $25.3^{b}$ & $27.3^{b}$ & $26.3^{b}$ & $24.2^{b}$ & $26.0^{b}$ & $25.1^{b}$ \\
\hline $\begin{array}{l}\mathrm{L}_{2}-\text { Cow urine @ } 500 \text { litres ha }^{-1} \text { at } 30, \\
60 \text { and } 90 \text { DAS }\end{array}$ & $11.7^{\circ}$ & $13.6^{b}$ & $12.7^{\circ}$ & $10.5^{c}$ & $12.0^{c}$ & $11.1^{c}$ & $24.1^{\circ}$ & $26.0^{\circ}$ & $25.1^{\circ}$ & $22.9^{\circ}$ & $24.7^{\circ}$ & $23.8^{\circ}$ \\
\hline $\begin{array}{l}\mathrm{L}_{3}-\text { Bio digester @ } 2500 \text { litres ha }{ }^{-1} \text { at } \\
30,60 \text { and } 90 \mathrm{DAS}\end{array}$ & $12.9^{\mathrm{a}}$ & $14.8^{\mathrm{a}}$ & $13.8^{\mathrm{a}}$ & $11.4^{\mathrm{a}}$ & $13.3^{\mathrm{a}}$ & $12.4^{\mathrm{a}}$ & $26.0^{\mathrm{a}}$ & $28.0^{\mathrm{a}}$ & $27.0^{\mathrm{a}}$ & $24.8^{\mathrm{a}}$ & $26.7^{\mathrm{a}}$ & $25.7^{\mathrm{a}}$ \\
\hline $\mathrm{L}_{4}-$ Control (no liquid manure application) & $11.3^{\mathrm{d}}$ & $13.2^{b}$ & $12.3^{\mathrm{d}}$ & $9.8^{\circ}$ & $11.7^{d}$ & $10.7^{d}$ & $23.6^{c}$ & $25.3^{d}$ & $24.4^{d}$ & $22.4^{c}$ & $24.0^{d}$ & $23.2^{d}$ \\
\hline S.Em \pm & 0.13 & 0.17 & 0.11 & 0.16 & 0.1 & 0.09 & 0.19 & 0.09 & 0.1 & 0.17 & 0.09 & 0.09 \\
\hline \multicolumn{13}{|l|}{ Interactions-(MXL) } \\
\hline$M_{1} L_{1}$ & $10.2^{j-1}$ & $12.2^{j-1}$ & $11.2^{\operatorname{lm}}$ & $8.7^{\mathrm{m}}$ & $10.6^{\mathrm{k}-\mathrm{m}}$ & $9.7^{k 1}$ & $21.7^{j}$ & $23.5^{\mathrm{i}}$ & $22.6^{h}$ & $20.5^{i}$ & $22.5^{\mathrm{j}}$ & $21.5^{\mathrm{h}}$ \\
\hline$M_{1} L_{2}$ & $9.9^{\mathrm{kl}}$ & $11.8^{k l}$ & $10.8^{\operatorname{lm}}$ & $8.3^{\mathrm{m}}$ & $10.2^{\operatorname{lm}}$ & $9.2^{\operatorname{lm}}$ & $20.3^{k}$ & $22.4^{j}$ & $21.3^{i}$ & $19.1^{j}$ & $21.2^{k}$ & $20.2^{i}$ \\
\hline$M_{1} L_{3}$ & $10.6^{i-k}$ & $12.7^{n-1}$ & $11.6^{j-1}$ & $9.2^{\mathrm{jm}}$ & $11.2^{\mathrm{jk}}$ & $10.2^{\mathrm{jk}}$ & $22.4^{\text {h-j }}$ & $24.5^{\mathrm{h}}$ & $23.4^{h}$ & $21.2^{g-i}$ & $23.1^{i}$ & $22.2^{h}$ \\
\hline $\mathrm{M}_{1} \mathrm{~L}_{4}$ & $9.5^{1}$ & $11.4^{\prime}$ & $10.5^{\mathrm{m}}$ & $8.01 \mathrm{~m}$ & $9.8^{\mathrm{m}}$ & $8.9^{\mathrm{m}}$ & $19.9^{k}$ & $21.7^{j}$ & $20.8^{i}$ & $18.7^{j}$ & $20.6^{k}$ & $19.7^{i}$ \\
\hline$M_{2} L_{1}$ & $11.2^{n-j}$ & $13.1^{g-k}$ & $12.2^{i-k}$ & $9.8^{i-1}$ & $11.7^{\mathrm{j}}$ & $10.7^{\mathrm{j}}$ & $23.3^{g-i}$ & $25.2^{9}$ & $24.3^{9}$ & $22.5^{\mathrm{fg}}$ & $23.9^{h}$ & $23.2^{9}$ \\
\hline $\mathrm{M}_{2} \mathrm{~L}_{2}$ & $10.5^{i-1}$ & $12.4^{i-1}$ & $11.5^{\mathrm{kl}}$ & $9.0^{k \cdot m}$ & $10.9^{k l}$ & $9.9^{k \mid}$ & $22.5^{h-j}$ & $24.3^{\mathrm{h}}$ & $23.4^{h}$ & $21.1^{\mathrm{hi}}$ & $22.8^{\mathrm{ij}}$ & $21.9^{h}$ \\
\hline $\mathrm{M}_{2} \mathrm{~L}_{3}$ & $11.7^{\text {th }}$ & $13.6^{e-i}$ & $12.7^{9-i}$ & $10.3^{0-k}$ & $12.2^{f-i}$ & $11.3^{-\mathrm{gi}}$ & $23.9^{f-h}$ & $25.8^{f_{9}}$ & $24.8^{f g}$ & $22.5^{\text {fg }}$ & $24.6^{9}$ & $23.6^{19}$ \\
\hline $\mathrm{M}_{2} \mathrm{~L}_{4}$ & $10.4^{j-1}$ & $12.2^{j-1}$ & $11.3^{l m}$ & $8.8^{\operatorname{lm}}$ & $10.6^{\mathrm{k}-\mathrm{m}}$ & $9.7^{k 1}$ & $22.1^{i j}$ & $24.0^{\text {hi }}$ & $23.1^{\mathrm{h}}$ & $20.7^{i}$ & $22.2^{j}$ & $21.5^{\mathrm{h}}$ \\
\hline $\mathrm{M}_{3} \mathrm{~L}_{1}$ & $12.3^{\mathrm{d}-g}$ & $14.3^{c-g}$ & $13.3^{\mathrm{d}-\mathrm{g}}$ & $10.9^{d-i}$ & $12.7^{d-j}$ & $11.8^{e-h}$ & $25.3^{d-f}$ & $27.4^{\mathrm{e}}$ & $26.3^{\mathrm{e}}$ & $24.1^{\text {de }}$ & $26.0^{\text {ef }}$ & $25.1^{\mathrm{e}}$ \\
\hline $\mathrm{M}_{3} \mathrm{~L}_{2}$ & $11.7^{\mathrm{fhh}}$ & $13.6^{e-j}$ & $12.6^{9-1}$ & $10.1^{h-k}$ & $12.0^{9 \cdot j}$ & $11.0^{\mathrm{hi}}$ & $23.9^{\text {f-h }}$ & $25.7^{f^{9}}$ & $24.8^{f g}$ & $22.7^{f}$ & $24.5^{\text {gh }}$ & $23.6^{f g}$ \\
\hline $\mathrm{M}_{3} \mathrm{~L}_{3}$ & $12.9^{c-e}$ & $14.8^{b-f}$ & $13.8^{c e}$ & $11.4^{\circ-9}$ & $13.3^{c e}$ & $12.4^{c e}$ & $26.1^{\text {cd }}$ & $28.3^{c d}$ & $27.2^{\text {cd }}$ & $25.1^{\mathrm{cd}}$ & $26.9^{\text {cd }}$ & $26.0^{\text {cd }}$ \\
\hline $\mathrm{M}_{3} \mathrm{~L}_{4}$ & $11.5^{g-i}$ & $13.3^{f \cdot j}$ & $12.4^{\mathrm{h}-\mathrm{j}}$ & $9.9 \mathrm{~h}^{-1}$ & $11.8^{h_{j-j}}$ & $10.8^{\mathrm{j}}$ & $23.2^{9-i}$ & $25.2^{9}$ & $24.2^{9}$ & $22.2^{\text {th }}$ & $24.1^{g h}$ & $23.1^{9}$ \\
\hline$M_{4} L_{1}$ & $12.6^{c-f}$ & $14.6^{b-9}$ & $13.6^{d-f}$ & $11.2^{\mathrm{dh}}$ & $13.1^{\text {cet }}$ & $12.1^{d-f}$ & $25.7^{c-e}$ & $27.8^{\text {de }}$ & $26.7^{\text {de }}$ & $24.5^{c e}$ & $26.3^{\text {de }}$ & $25.4^{\mathrm{de}}$ \\
\hline $\mathrm{M}_{4} \mathrm{~L}_{2}$ & $12.0^{\mathrm{e}-\mathrm{h}}$ & $13.9^{\mathrm{d}-i}$ & $12.9^{f-1}$ & $10.4^{f-j}$ & $12.3^{f-i}$ & $11.3^{g-i}$ & $24.4^{e-g}$ & $26.3^{f}$ & $25.3^{f}$ & $23.2^{\text {ef }}$ & $24.8^{9}$ & $24.0^{t}$ \\
\hline$M_{4} L_{3}$ & $13.2^{b-d}$ & $15.0^{b-e}$ & $14.1^{\text {cd }}$ & $11.7^{b-e}$ & $13.5^{\text {od }}$ & $12.6^{\mathrm{cd}}$ & $26.2^{\text {cd }}$ & $28.3^{\text {cd }}$ & $27.3^{\mathrm{cd}}$ & $25.1^{\text {cd }}$ & $26.9^{\text {cd }}$ & $26.0^{\text {cd }}$ \\
\hline $\mathrm{M}_{4} \mathrm{~L}_{4}$ & $11.8 e^{-h}$ & $13.7^{\mathrm{d}-\mathrm{i}}$ & $12.8^{f-i}$ & $10.3^{9-k}$ & $12.2^{f-i j}$ & $11.2^{\mathrm{hi}}$ & $23.9^{\text {th }}$ & $25.6^{i g}$ & $24.7^{i g}$ & $22.6^{f}$ & $24.5^{\mathrm{gh}}$ & $23.5^{f g}$ \\
\hline $\mathrm{M}_{5} \mathrm{~L}_{1}$ & $13.7^{b c}$ & $15.6^{a-c}$ & $14.6^{\mathrm{bc}}$ & $12.1^{b-d}$ & $14.0^{\mathrm{bc}}$ & $13.0^{\text {bc }}$ & $27.7^{\mathrm{ab}}$ & $29.6^{b}$ & $28.7^{b}$ & $26.6^{\mathrm{ab}}$ & $28.5^{\mathrm{b}}$ & $27.5^{b}$ \\
\hline$M_{5} L_{2}$ & $13.2^{b-d}$ & $14.7^{b-f}$ & $13.9^{c e}$ & $11.6^{b-f}$ & $13.2^{c e t}$ & $12.4^{c-e}$ & $26.9^{b c}$ & $28.7^{\circ}$ & $27.8^{c}$ & $25.5^{b-d}$ & $27.4^{c}$ & $26.5^{c}$ \\
\hline $\mathrm{M}_{5} \mathrm{~L}_{3}$ & $14.1^{\mathrm{ab}}$ & $16.0^{\mathrm{ab}}$ & $15.1^{\mathrm{ab}}$ & $12.7^{\mathrm{ab}}$ & $14.5^{\mathrm{ab}}$ & $13.6^{\mathrm{ab}}$ & $28.4^{\mathrm{a}}$ & $30.4^{\mathrm{a}}$ & $29.4^{\mathrm{ab}}$ & $27.2^{\mathrm{a}}$ & $29.1^{\mathrm{ab}}$ & $28.2^{\mathrm{ab}}$ \\
\hline $\mathrm{M}_{5} \mathrm{~L}_{4}$ & $12.2^{\mathrm{d}-\mathrm{h}}$ & $14.1^{c-h}$ & $13.2^{e-h}$ & $10.7^{-\mathrm{ei}}$ & $12.6^{\mathrm{eh}}$ & $11.6^{\mathrm{fhh}}$ & $26.2^{\text {cd }}$ & $27.1^{\mathrm{e}}$ & $26.7^{\mathrm{de}}$ & $25.1^{\mathrm{cd}}$ & $25.6^{f}$ & $25.4^{\mathrm{de}}$ \\
\hline$M_{6} L_{1}$ & $14.1^{\mathrm{ab}}$ & $16.0^{\mathrm{ab}}$ & $15.0^{\mathrm{ab}}$ & $12.6^{a-c}$ & $14.5^{\mathrm{ab}}$ & $13.6^{\mathrm{b}}$ & $28.2^{\mathrm{ab}}$ & $30.4^{\mathrm{a}}$ & $29.3^{\mathrm{ab}}$ & $27.0^{\mathrm{a}}$ & $28.9^{b}$ & $27.9^{b}$ \\
\hline $\mathrm{M}_{6} \mathrm{~L}_{2}$ & $13.2^{b-d}$ & $15.1^{b-d}$ & $14.2^{\text {cd }}$ & $11.6^{b-f}$ & $13.6^{\text {cd }}$ & $12.6^{\text {cd }}$ & $26.8^{\mathrm{bc}}$ & $28.7^{\circ}$ & $27.8^{c}$ & $25.7^{\mathrm{bc}}$ & $27.3^{\mathrm{c}}$ & $26.5^{\circ}$ \\
\hline $\mathrm{M}_{6} \mathrm{~L}_{3}$ & $14.8^{\mathrm{a}}$ & $16.7^{\mathrm{a}}$ & $15.7^{\mathrm{a}}$ & $13.3^{\mathrm{a}}$ & $15.2^{\mathrm{a}}$ & $14.3^{\mathrm{a}}$ & $28.9^{\mathrm{a}}$ & $30.9^{\mathrm{a}}$ & $29.9^{\mathrm{a}}$ & $27.8^{\mathrm{a}}$ & $29.5^{\mathrm{a}}$ & $28.7^{\mathrm{a}}$ \\
\hline $\mathrm{M}_{6} \mathrm{~L}_{4}$ & $12.7^{c-f}$ & $14.6^{b-9}$ & $13.6^{\mathrm{d}-\mathrm{f}}$ & $11.0^{d-i}$ & $12.9^{d-f}$ & $12.0^{\mathrm{d}-g}$ & $26.2^{\text {cd }}$ & $28.2^{c d}$ & $27.2^{\mathrm{cd}}$ & $25.1^{\text {cd }}$ & $26.9^{\text {cd }}$ & $26.0^{\text {cd }}$ \\
\hline S.Em \pm & 0.32 & 0.43 & 0.27 & 0.39 & 0.25 & 0.23 & 0.47 & 0.23 & 0.26 & 0.21 & 0.22 & 0.23 \\
\hline Initial & \multicolumn{6}{|c|}{7.7} & \multicolumn{6}{|c|}{17.2} \\
\hline
\end{tabular}

Means followed by the same alphabet do not differ significantly by DMRT $(0.05)$ 
60 and 90 DAS $\left(\mathrm{M}_{6} \mathrm{~L}_{3}\right)$ recorded significantly higher dehydrogenase (15.7 and $14.3 \mathrm{~g}_{\text {TPF }} \mathrm{g}^{-1}$ soil day ${ }^{-1}$ ) and phosphatase (29.9 and $28.7 \mathrm{~g} \mathrm{pNP} \mathrm{g}^{-1}$ soil h$^{-1}$ ) at flowering and harvest respectively.

Addition of organic amendments and adoption of management practices that increase soil organic matter lead to increased enzyme activity. Plant roots stimulate enzyme activity because of their positive effect on microbial activity and production of exudates rich in substrates acted on by enzymes. Enzyme activity in soil increased under organic nutrient management practice when compared to inorganic nutrient management practice due to continuous addition of organic manures in the form of FYM, vermicompost, green manure and bio-fertilizers. The higher dehydrogenase activity after addition of organic manure could be due to increased microbial population, which is known to stimulate the dehydrogenase activity in soil (Watts et al., 2010). Tejada et al. (2010) reported higher dehydrogenase enzyme activity in soil due to application of vermicompost obtained from cow dung continuously for three years. This was attributed to greater labile fraction of organic matter. The maximum dehydrogenase activity was recorded with 100 per cent substitution of RDN with vermicompost, poultry manure, biogas slurry and FYM. Incorporation of green manures enhanced soil enzyme activity (Ramalingam and Kannaiyan, 2006). Similar results were observed by Mishra et al. (2008). The maximum phosphatase activity in soil under organic nutrient management practice due to incorporation of organic manures that increase decomposition process thereby increase the microbial activity. The higher phosphatase activity was recorded (Meena et al., 2014) with 100 per cent substitution of RDN with concentrate organic manure (wellgrow formulations). Sriramachandrasekharan and Ravichandran (2011) reported that addition of organic substances to the soil served as a carbon source that enhanced microbial biomass and phosphatase activity, showing that these enzymes are of microbiological origin (Bohem etal., 2005).

Based on the results, it can be concluded that higher soil microbial dynamics viz., $\mathrm{N}$ fixers, PSB, Azospirillum spp. population and soil enzymes such as dehydrogenase and phosphatase activity at flowering and harvest was enhanced with integrated application of FYM (1/3) + vermicompost (1/3) + green leaf manure (1/3) equivalent to RDN of $100 \mathrm{~kg} \mathrm{ha}^{-1}$ with recommended FYM (5 t ha ${ }^{-1}$ ) and microbial consortium (Azospirillum + PSB) with soil application of biodigester (2500 I ha' $\left.{ }^{1}\right)$ in aerobic rice.

\section{Acknowledgment}

The Authors are extremely thankful to the Department of Agricultural Microbiology, College of Agriculture, UAS, Dharwad for providing laboratory facilities for the research.

\section{References}

Aon, M.A. and A.C. Colaneri: Temporal and spatial evolution of enzymatic activities and physico-chemical properties in an agricultural soil. Appl. Soil Ecol., 18, 255-270 (2001).

Bohem, L., U. Langer and F. Bohem: Microbial biomass, enzyme activities and microbial community structure in two European longterm field experiments. Agric. Ecosyst. Environ., 109, 141-152 (2005).

Buenaventurada, C., P. and Y. Tokiwa: Production of D-lactic acid from sugarcane molasses, sugarcane juice and sugar beet juice by Lactobacillus delbrueckii. Biotechnol. Lett., 29, 1329-1332 (2007).

Casida, L. E., D. A. Klein and T. Santoro: Soil dehydrogenase activity. Soil Sci., 98, 371-376 (1964).

Cochran, W. G.: Estimation of bacterial density by mean of most probable number. Biometrics, 6, 105-116 (1950).

Efsun, D., F.O.T. Sagban and H.S. Baskaya: Evaluation of soil enzyme activities as soil quality indicators insludge-amended soils. J. Environ. Biol., 36, 919-926 (2015).

Evasi, Z. and M. A. Tabatabai: Phosphatase in soils. Soil Biol. Biochem., 9, 167-172(1979).

Gomez, K.A. and A.A. Gomez: Statistical Procedures for Agricultural Research. $2^{\text {nd }}$ Edn., John Wiley \& Sons, New York (1984).

Iswandi, A.O.P., T.M. Rupela and Thiyagarajan: A review of studies on SRI effects on beneficial organisms in rice soil rhizospheres. Paddy Water Environ., 9, 53-64 (2011).

Jackson, L. E., T.M. Bowles, A.K. Hodson and C. Lazcano: Soil microbialroot and microbial rhizosphere processes to increase nitrogen availability and retentionin agroecosystems. Curr. Opin. Environ. Sustain., 4, 517-522 (2012).

Kallenbach, C. and A.S. Grandy: Control over soil microbial biomass responses to carbon amendments in agricultural systems: A meta-analysis. Agric. Ecosyst. Environ., 144, 241-252 (2011).

Majumdar, D., M. Patel, R. Darbar and M. Vyas: Short-term emissions of ammonia and carbon dioxide from cattle urine contaminated tropical grassland microcosm. Environ. Monitor. Assess., 122, 9$25(2006)$

Meena, V. S., B. R. Maurya, R. S. Meena, S. K. Meena, N. P. Singh, V. K. Malik, V. Kumar and L. K. Jat: Microbial dynamics as influenced by concentrate manure and inorganic fertilizer in alluvium soil of Varanasi, India. Africian J. Microbiol. Res., 8, 257-263(2014).

Mishra, B., J. Prasad, A. Sharma, B.P. Singh and S.K. Singh: Influence of continuous application of amendments to maize-wheat cropping system on dynamics of soil microbial biomass in Alfisols of Jharkhand. J. Indian Soc. Soil Sci., 56, 71-75(2008).

Moeskops, B., D. Buchan, S. Sleutel, L. Herawaty, E. Husen, R,Saraswati, D. Setyorini and S. De Neve: Soil microbial communities and activities under intensive organic and conventional vegetable farming in West Java, Indonesia. Appl. Soil Ecol., 45, 112-120 (2010).

Mondal, S.S., S. Sarkar, A. Ghosh and J. Das: Response of summer rice (Oryza sativa L.) to different organic and inorganic sources of nutrients. Crop Res., 25, 219-222(2003).

Ponmurugan, P. and C. Gopi: In vitro production of growth regulators and phosphatase activity by phosphate solubilizing bacteria. African J. Biotechol., 5, 348-350 (2006).

Ramalingam, N. and S. Kannaiyan: Impact of green manures and sulphur application on soil enzyme activity and grain yield of rice. Mysore J. Agric. Sci., 40, 68-73(2006). 
Saini, V.K., S.C. Bhandari and J. Panwar: Establishment and influence of microbial inoculants along with FYM and fertilizer on sorghum (Sorghum bicolorL.). Indian J. Microbiol., 45, 147-150 (2005).

Shiling, C., Y. Weiwei, Z. Zhi and L. Surong: Soil properties and enzyme activities as affected by biogas slurry irrigation in Three Gorges Reservoir areas of China. J. Environ. Biol., 36, 513-520 (2015).

Sriramachandrasekharan, M.V. and M. Ravichandran: Enzyme dynamics and soil properties in paddy soil fertilized with mineral and green manure sources. Agric. Segm., 2, 1553-1558 (2011).

Stursova, M. and P. Baldrian: Effects of soil properties and management on the activity of soil organic matter transforming enzymes and the quantification of soil-bound and free activity. Plant and Soil, $\mathbf{3 3 8 ,}$ 99-110 (2010).
Sudhanshu, S.K., M. Joshi and S. Bhaskar: Characterization of farmers jeevamrutha formulation with respect to aerobic rice. Mysore $\mathrm{J}$. Agric. Sci., 43, 570-573 (2009).

Taylor, J.P., B. Wilson, M.S. Mills and R.G. Burns: Comparison of microbial number and enzymatic activities in surface soils and subsoil using various techniques. Soil Biol. Biochem., 34, 387401(2002).

Tejada, M., I.H. Gomez, Teresa and C. Garcia: Utilization of vermicompost in the soil restoration: Effects on soil biological properties. Soil Sci. Soc. American J., 74, 525-532 (2010).

Watts, D.B., T.H. Allen, Y. Feng and S.A. Prior: Soil microbial community dynamics as influenced by composted dairy manure, soil properties, and landscape position. Soil Sci., 175, 474-486 (2010). 